\title{
AUTOMORPHISMS OF THE SEMIGROUP OF ALL DIFFERENTIABLE FUNCTIONS
}

\author{
by KENNETH D. MAGILL, JR
}

(Received 7 March, 1966)

1. Introduction. Let $R$ denote the space of real numbers and let $D(R)$ denote the family of all functions mapping $R$ into $R$ that are (finitely) differentiable at each point of $R$. Since the composition $f \circ g$ of two differentiable functions is also differentiable and since the composition operation is associative, it follows that $D(R)$ is a semigroup with this operation. Such semigroups have been studied previously. Nadler, in [4], has shown that the semigroup of al differentiable functions mapping the closed unit interval into itself has no idempotent elements other than the identity function and the constant functions. The proof of that result carries over easily to the semigroup $D(R)$. As the title indicates, the purpose of this paper is to investigate the automorphisms of $D(R)$. Our principal result states that every automorphism of $D(R)$ is inner. By applying this result, we obtain three corollaries. The first states that the automorphism group of $D(R)$ is isomorphic to the group (under composition) of all strictly monotonic functions mapping $R$ onto $R$ that have a finite, nonzero derivative at each point of $R$. The second corollary states that each automorphism of $D(R)$ has a unique extension to an automorphism of $S(R)$, the semigroup of all continuous functions mapping $R$ into $R$. The third and last corollary states that the automorphism group of the near-ring of all differentiable functions on $R$ is isomorphic to the multiplicative group of all nonzero real numbers.

Finally, we take this opportunity to acknowledge our indebtedness to J. T. Rosenbaum for his valuable suggestions.

2. Theorems and corollaries. Before proving the main result, we recall some definitions and discuss some notations that will be used. First, let us recall that an element $z$ of a semigroup $S$ is said to be a left zero if $z a=z$ for each $a$ in $S$. It is immediate that the set of all left zeros of a semigroup is an ideal of that semigroup. Now, for each real number $x$, we use the symbol $\mathbf{x}$ to denote the constant function in $D(R)$ which maps every real number into $x$, i.e., $\mathbf{x}(y)=x$ for each $y$ in $R$. One shows easily that the set of constant functions of $D(R)$ is precisely the ideal of left zeros of $D(R)$. Finally, we recall that an automorphism $\phi$ of $D(R)$ is said to be inner if there exists an element $h$ in $D(R)$ having a two-sided inverse $h^{-1}$ such that $\phi(f)=h \circ f \circ h^{-1}$ for each $f$ in $D(R)$. With this in mind, we are ready to prove

THEOREM 2.1. Every automorphism of $D(R)$ is inner.

Proof. Let $\phi$ be any automorphism of $D(R)$ and let $x$ be any real number. Then $\mathbf{x}$ is a left zero of $D(R)$ and hence $\phi(\mathbf{x})$ must also be a left zero of $D(R)$. This means that $\phi(\mathbf{x})=\mathbf{y}$ for some real number $y$. Define a mapping $h$ from $R$ into $R$ by $h(x)=y$. Since $\phi$ maps the ideal of left zeros of $D(R)$ bijectively onto itself, it follows that $h$ is a bijection from $R$ onto $R$. Using the fact that for any $x$ in $R, \phi(\mathbf{x})=\mathbf{h}(\mathbf{x})$, we see that for $f$ in $D(R)$ and $y$ in $R$, 


$$
\begin{aligned}
\left(h \circ f \circ h^{-1}\right)(y)=h\left(f\left(h^{-1}(y)\right)\right)=\mathbf{h}\left(\mathbf{f}\left(\mathbf{h}^{-1}(\mathbf{y})\right)\right)(y)=\phi\left(\mathbf{f}\left(\mathbf{h}^{-1}(\mathbf{y})\right)\right)(y) & =\phi\left(f \circ \mathbf{h}^{-1}(\mathbf{y})\right)(y) \\
= & \left(\phi(f) \circ \phi\left(\mathbf{h}^{-1}(\mathbf{y})\right)(y)=(\phi(f) \circ \mathbf{y})(y)=\phi(f)(y) .\right.
\end{aligned}
$$

Thus $\phi(f)=h \circ f \circ h^{-1}$.

Now, let $x_{0}$ be any point of $R$. We want to show that $h$ is continuous at $x_{0}$, so let $\varepsilon>0$ be given. Choose a number $y_{0} \neq h\left(x_{0}\right)$ and let $g$ be any function in $D(R)$ with the properties:

$$
g(x)=h\left(x_{0}\right) \text { for } x \leqq h\left(x_{0}\right)-\varepsilon
$$

and for $x \geqq h\left(x_{0}\right)+\varepsilon$,

$$
g\left(h\left(x_{0}\right)\right)=y_{0} .
$$

Then, since $\phi$ is an automorphism, there exists a function $f$ in $D(R)$ such that $\phi(f)=g$. Suppose that $f\left(\mathbf{x}_{0}\right)=\mathbf{x}_{0}$. Then $f \circ x_{0}=x_{0}$, which implies that

$$
\mathbf{h}\left(\mathbf{x}_{0}\right)=\phi\left(\mathbf{x}_{0}\right)=\phi\left(f \circ \mathbf{x}_{0}\right)=\phi(f) \circ \phi\left(\mathbf{x}_{0}\right)=g \circ \mathbf{h}\left(\mathbf{x}_{0}\right)
$$

which, in turn, implies that $g\left(h\left(x_{0}\right)\right)=h\left(x_{0}\right)$, which contradicts (2.1.2). Thus $f\left(x_{0}\right) \neq x_{0}$, and since $f$ is continuous at $x_{0}$, there exists a positive number $\delta$ such that $f(x) \neq x_{0}$ if $\left|x-x_{0}\right|<\delta$. But this means that $f \circ \mathbf{x} \neq \mathbf{x}_{0}$ which implies that $\phi(f \circ \mathbf{x}) \neq \phi\left(\mathbf{x}_{0}\right)$, since $\phi$ is a bijection. Hence $\phi(f) \circ \mathbf{h}(\mathbf{x}) \neq \mathbf{h}\left(\mathbf{x}_{0}\right)$, which implies that $g(h(x))=\phi(f)(h(x)) \neq h\left(x_{0}\right)$. Therefore it follows from (2.1.1) that $\left|h(x)-h\left(x_{0}\right)\right|<\varepsilon$ if $\left|x-x_{0}\right|<\delta$. This proves that $h$ is continuous at the point $x_{0}$ and thus at every point of $R$, since the choice of $x_{0}$ was arbitrary. This, together with the fact that $h$ is a bijection, allows us to conclude that $h$ must be either strictly increasing or strictly decreasing. Then $h^{-1}$ is also a continuous function which is strictly increasing or strictly decreasing.

Now we want to show that $h$ is differentiable at any given point $x_{0}$ of $R$. Theorem 4, page 211 , of [5] states that any monotonic function defined on a closed interval $[a, b]$ has a finite derivative at almost all points of $[a, b]$. From this we conclude that there exists a point $x_{1}$ of $R$ at which $h$ has a finite derivative. Let $h\left(x_{1}\right)=x_{2}$ and define two functions $f$ and $t$ by

and

$$
\begin{aligned}
& f(x)=x+x_{0}-x_{1} \\
& t(x)=h\left(x+x_{1}\right)-x_{2} .
\end{aligned}
$$

Since $h$ is a homeomorphism from $R$ onto $R$, the function $t$ is also. This, together with the fact that $t(0)=0$, implies that $t(x) \neq 0$ if $x \neq 0$ and also that $t(x) \rightarrow 0$ when $x \rightarrow 0$. Now we observe that $f \in D(R)$ and thus $g=\phi(f)=h \circ f \circ h^{-1} \in D(R)$. With some calculation, one shows that for $x \neq 0$,

$$
\frac{h\left(x_{0}+x\right)-h\left(x_{0}\right)}{x}=\frac{g\left(x_{2}+t(x)\right)-g\left(x_{2}\right)}{t(x)} \cdot \frac{h\left(x_{1}+x\right)-h\left(x_{1}\right)}{x} .
$$

Since $g$ is differentiable at every point of $R$ and $h$ is differentiable at $x_{1}$, it follows that $h$ is differentiable at $x_{0}$. Indeed $h^{\prime}\left(x_{0}\right)=g^{\prime}\left(x_{2}\right) h^{\prime}\left(x_{1}\right)$. Since the point $x_{0}$ was chosen arbitrarily we conclude that $h$ belongs to $D(R)$. Finally, let us note that the automorphism $\phi^{-1}$ is given 
by $\phi^{-1}(f)=h^{-1} \circ f \circ h$ for each $f$ in $D(R)$ and hence that the previous argument given for $h$ now applies to $h^{-1}$. Therefore $h^{-1}$ also belongs to $D(R)$. This completes the proof that $\phi$ is an inner automorphism.

Now let $G$ denote the group (under composition) of all strictly monotonic functions mapping $R$ onto $R$ that have a finite, nonzero derivative at each point of $R$ and let $\mathfrak{U}$ denote the automorphism group of $D(R)$. The previous theorem states that for each $\phi \in \mathfrak{U}$, there exists an element $h$ in $D(R)$ with a two-sided inverse $h^{-1}$ (also in $D(R)$ ) such that $\phi(f)=h \circ f \circ h^{-1}$ for each $f$ in $D(R)$. The fact that $h^{-1}$ has a finite derivative everywhere implies that $h^{\prime}(x)$ is never zero. It follows that $h$ belongs to $G$, since a continuous bijection from $R$ onto $R$ must be strictly monotonic. The function $h$ is uniquely determined by $\phi$ and hence we may define a mapping $\Phi$ from $\mathfrak{Z}$ into $G$ by $\Phi(\phi)=h$. It is a routine matter to verify that $\Phi$ is a homomorphism. In fact, $\Phi$ is an epimorphism, since for any $k \in G$, the mapping $\psi$ defined by $\psi(f)=k \circ f \circ k^{-1}$ is an automorphism of $D(R)$ and $\Phi(\psi)=k$. Finally, if $\Phi(\phi)$ is the identity mapping, then $\phi$ must be the identity automorphism, i.e., the kernel of $\Phi$ consists of the identity of $\mathfrak{A}$. Thus $\Phi$ is an isomorphism and we have proved

COROLlaRY 2.2. The automorphism group of $D(R)$ is isomorphic to the group of all strictly monotonic functions mapping $R$ onto $R$ that have a finite, nonzero derivative at each point of $R$.

Now let $S(R)$ denote the semigroup of all continuous functions mapping $R$ into $R$. Evidently, $D(R)$ is a subsemigroup of $S(R)$. Let $\phi$ be any automorphism of $D(R)$ and again let $h$ be the function in $D(R)$ such that $\phi(f)=h \circ f \circ h^{-1}$ for each $f$ in $D(R)$. It is easily verified that the mapping $\phi^{*}$ from $S(R)$ into $S(R)$ defined by

$$
\phi^{*}(f)=h \circ f \circ h^{-1} \text { for each } f \text { in } S(R)
$$

is an automorphism of $S(R)$ that is an extension of $\phi$. Suppose that $\psi$ is any other automorphism of $S(R)$ that is an extension of $\phi$. Then by statement (3.5) of [3], it follows that there exists a homeomorphism $k$ of $R$ such that $\psi(f)=k \circ f \circ k^{-1}$ for each $f$ in $S(R)$. Then, for any real number $x$,

$$
\mathbf{h}(\mathbf{x})=\phi(\mathbf{x})=\psi(\mathbf{x})=\mathbf{k}(\mathbf{x}) .
$$

Hence, $h(x)=k(x)$ for each $x$ and it follows that $\psi=\phi^{*}$. This proves

COROLlARY 2.3. Every automorphism of $D(R)$ has a unique extension to an automorphism of $S(R)$.

Before stating Corollary 2.4 , let us recall that a near-ring is a triple, $(T,+, o)$, where $(T,+)$ is a group, $(T, \circ)$ is a semigroup and multiplication is right distributive with respect to addition. $D(R)$ is a near-ring relative to pointwise addition and composition. We conclude with the following result which concerns the automorphism group of this near-ring.

COROLlaRY 2.4. The automorphism group of the near-ring $D(R)$ is isomorphic to the multiplicative group of nonzero real numbers.

Proof. Denote the automorphism group of the near-ring $D(R)$ by 27 and let $\phi$ be an element of $2 \pi$. Since $\phi$ is an automorphism of the semigroup $(D(R), 0)$, it follows from Theorem 
2.1 that there exists a function $h$ in $D(R)$ with the property that, for any real number $x$, $\phi(\mathbf{x})=\mathbf{h}(\mathbf{x})$. Then, for any two real numbers $x$ and $y$, we have

$$
\mathbf{h}(\mathbf{x}+\mathbf{y})=\phi(\mathbf{x}+\mathbf{y})=\phi(\mathbf{x})+\phi(\mathbf{y})=\mathbf{h}(\mathbf{x})+\mathbf{h}(\mathbf{y}) .
$$

This implies that $h(x+y)=h(x)+h(y)$. Thus $h$ is both continuous and additive, and it is well known that there exists a real number $a$ such that

$$
h(x)=a x \text { for each } x \text { in } R .
$$

In this case, moreover, $a$ cannot be zero, since $h$ is a bijection. The number $a$ is uniquely determined by $\phi$ and so we may define a mapping $\Phi$ from 27 into the set of nonzero real numbers by $\Phi(\phi)=a$. For any nonzero real number $b$, let $\phi_{b}$ denote the mapping from $D(R)$ into $D(R)$ that is defined by

$$
\left(\phi_{b}(f)\right)(x)=b f(x / b) .
$$

One shows in a straightforward manner that $\phi_{b}$ is an automorphism of $D(R)$ and that $\Phi\left(\phi_{b}\right)=b$. Thus $\Phi$ maps 27 onto the multiplicative group of all nonzero real numbers. Since it is a routine matter to show that $\Phi$ is a homomorphism, the details will not be given. To see that $\Phi$ is actually an isomorphism, suppose that $\Phi(\phi)=1$. Then, according to (2.4.1), the mapping $h$ that is determined by $\phi$ is given by $h(x)=x$ for each real number $x$, i.e., $h$ is the identity mapping on $R$, which implies that $\phi$ is the identity mapping on $D(R)$. Hence the kernel of $\Phi$ consists of the identity, and it follows that $\Phi$ is an isomorphism from $2 \pi$ onto the multiplicative group of all nonzero real numbers.

\section{REFERENCES}

1. A. H. Clifford and G. B. Preston, The algebraic theory of semigroups, Amer. Math. Soc. Math. Surveys, No. 7, (1961).

2. E. S. Ljapin, Semigroups, Translations of Mathematical Monographs, Vol. 3, Amer. Math. Soc., (1963). 984-988.

3. K. D. Magill, Jr, Semigroups of continuous functions, Amer. Math. Monthly 71 (1964),

4. S. B. Nadler, Jr, The idempotents of a semigroup, Amer. Math. Monthly 70 (1963), 996-997

5. I. P. Natanson, Theory of functions of a real variable, Vol. I, Frederick Ungar Publishing Co. (New York, 1955).

State UNIVERSity OF NeW YORK

BUFFALO 